\title{
Prácticas innovadoras en educación ciudadana. ¿Qué dicen las revistas académicas españolas?
}

\author{
Innovative practices in civic education. \\ What do Spanish academic journals say?
}

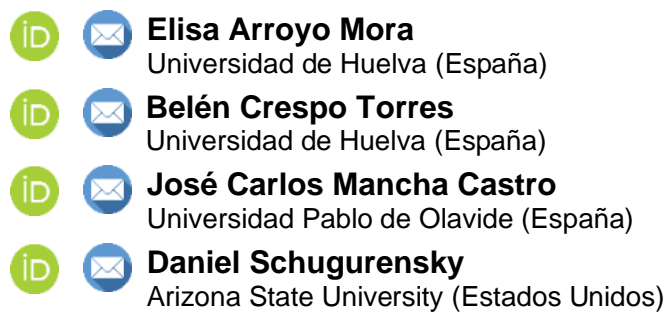

\section{Resumen}

En este trabajo se presentan resultados de un estudio de revisión bibliométrica y bibliográfica sobre experiencias innovadoras en educación ciudadana en los últimos diez años (2009-2018), extraídos de un total de 479 artículos publicados en cuatro revistas académicas españolas. Para el análisis de los datos se elaboró una ficha para clasificar la información de todos los artículos que fueron seleccionados. Los resultados reflejan la escasez de investigaciones referidas a la educación ciudadana y, particularmente, a experiencias de enseñanza-aprendizaje en esta área. A partir de la información recabada, y contrastándola con la base teórica y legislativa en torno a la formación de la ciudadanía participativa desde la escuela, se proponen una serie de recomendaciones didácticas con el fin de establecer pautas para abordar estas cuestiones en la educación formal y no formal y fomentar en el alumnado actitudes críticas y democráticas en su comunidad.

\begin{abstract}
This paper presents the results of a bibliometric and bibliographical review of innovative experiences in civic education in the last ten years (2009-2018) from a total of 479 articles published in four Spanish academic journals. The data were analysed according to specific categories. The results reflect the scarcity of research related to civic education and particularly teaching-learning experiences related to the subject of civic education. Based on the information gathered, and contrasting it with the theoretical and legislative basis around the formation of participatory citizenship at school, a number of recommendations are proposed in order to establish guidelines for addressing these issues in formal and nonformal education and to encourage critical and democratic attitudes in students.
\end{abstract}

\section{Palabras clave / Keywords}

Citizenship education, democratic participation, innovative experiences.

Educación ciudadana, participación democrática, experiencias innovadoras. 


\section{La educación para la ciudadanía: estado de la cuestión}

La asignatura "Educación para la Ciudadanía" (EC) fue introducida en el marco del sistema educativo oficial de España como materia curricular en la educación obligatoria en el año 2006, con la Ley Orgánica de Educación (LOE), por parte del gobierno socialista. Este hecho estuvo caracterizado por una intensa polémica y por la oposición de una parte de la sociedad, de algunos partidos políticos y de ciertas instituciones (como la Iglesia católica), que consideraban la materia como un instrumento de adoctrinamiento socialista al alumnado. Sin embargo, la aparición de esta materia en el currículo se originó con una recomendación del Consejo de Europa donde se afirmaba que la educación para la ciudadanía democrática era esencial para la misión principal del Consejo: promover una sociedad libre, tolerante y justa, que defendiera los valores y principios de libertad, pluralismo y derechos humanos. De esta forma, tal y como citan Cordero y Aguado (2015), en el preámbulo de la LOE se explicita el argumento para la introducción de esta nueva materia de la siguiente manera:

\section{La educación es el medio más adecuado para garantizar el ejercicio de la ciudadanía democrática, responsable, libre y crítica, que resulta indispensable para la constitución de sociedades avanzadas, dinámicas y justas.}

No obstante, el cambio de gobierno y la consiguiente reforma educativa materializada en la Ley Orgánica para la Mejora de la Calidad Educativa (LOMCE, 2013), del Partido Popular, provocó la supresión de la educación para la ciudadanía como materia íntegra para pasar a ser transversal, dándole el matiz de valores éticos y cívicos, justificando que deberían estar presentes en todas las áreas curriculares. En esta nueva legislación, se introdujeron una serie de competencias básicas que deberían garantizarse durante las diferentes etapas educativas, siendo estas de mayor o menor complejidad y abstracción en función de la edad y el desarrollo madurativo y cognitivo del alumnado.

Así, en el Real Decreto 126/2014 de 28 de febrero, en el que se establece el currículo básico de la Educación Primaria, la educación para la ciudadanía aparece como objetivo general de la etapa así como en el área de Ciencias Sociales, cuya finalidad última es conseguir la transmisión y puesta en práctica de valores que favorezcan la libertad personal, la responsabilidad, la ciudadanía democrática, la solidaridad, la tolerancia, la igualdad, el respeto y la justicia, considerando el paso del alumno por el sistema educativo como la preparación para el ejercicio de la ciudadanía y para la participación en la vida económica, social y cultural, con actitud crítica y responsable. Del mismo modo, en el Real Decreto 1105/2014 de 26 de diciembre, en el cual se establece el currículo básico de la Educación Secundaria Obligatoria y del Bachillerato, la competencia social y ciudadana se entiende como el ejercicio de una ciudadanía activa e integradora que exige el conocimiento y comprensión de los valores en que se asientan los estados y sociedades democráticas, de sus fundamentos, modos de organización y funcionamiento. Según lo establecido en el Boletín Oficial del Estado, esta competencia supone comprender la realidad social en que se vive, afrontar la convivencia y los conflictos, empleando el juicio ético basado en los valores y prácticas democráticas, y ejercer la ciudadanía, actuando con criterio propio, contribuyendo a la construcción de la paz y la democracia, y manteniendo una actitud constructiva, solidaria y responsable ante el cumplimiento de los derechos y obligaciones cívicas.

El objetivo principal de la educación ciudadana es educar a ciudadanos activos, capaces de crear un ambiente de paz en su sociedad y críticos ante las injusticias sociales de su comunidad (Morales Vargas, 2020). Sin embargo, según Cotton (2001), los contenidos que se proponen en las aulas en relación con la educación para la ciudadanía se presentan desconectados de los problemas reales a los que se enfrentan los estudiantes, lo que desencadena su pérdida de interés e implicación. Incluso con la presencia de una materia específica en cuestiones de ciudadanía en gobiernos anteriores, ya se advertía que, como sostiene GarcíaPérez (2009), no había un planteamiento claro del modelo de ciudadano que se perseguía, teniendo este el sesgo de una visión occidental y androcéntrica de los derechos humanos y del sistema de democracia política, y presentándose los derechos ciudadanos como algo dado y otorgado más que como algo conquistado, promoviendo una idea de ciudadano como consumidor, que debe acatar y respetar toda norma, más que como ciudadano activo.

Aunque la educación ciudadana que desde Europa se insta debe estar orientada a construir una ciudadanía global, que fomente la adquisición de competencias interculturales, basada en una participación crítica y democrática (Morales Vargas, 2020), la idea de participación no es un contenido relevante entre los saberes escolares convencionales y, por ello, su ubicación en la cultura escolar es dificultosa. De hecho, existe también una dificultad estructural del propio marco escolar, en cuanto a tiempos y espacios y con las 
restricciones a la expansión del conocimiento que imponen las tradiciones curriculares, que afecta de manera decisiva al aprendizaje real de los alumnos y a la actuación profesional de los profesores (García-Pérez y De Alba, 2012). Asimismo, su incorporación como materia a un currículum ya sobrecargado y con una lógica centrada en la información conceptual, con contenidos que resultan excesivamente teóricos, fríos y desvitalizados, provoca que ese conocimiento, tal y como sostienen García-Pérez (2009) y Mancha Castro (2019), no sea útil para entender los problemas reales y para intervenir en el mundo en el que viven los alumnos.

Guanipa, Albites, Aldana y Colina (2019) afirman que la ciudadanía debe construirse a través de la participación y el trabajo en grupo en pos de objetivos comunes, llevándose a cabo determinados proyectos bajo un régimen democrático que enseñe a respetar las diferencias y compartir los bienes y las oportunidades en conexión con la vida social y política de los estudiantes, cuestión que en muchas ocasiones no se da en las escuelas.

De la descontextualización del alumnado de los problemas reales en materia de democracia y participación se puede vislumbrar que el tipo de ciudadano que el sistema educativo pretende formar tal vez no se corresponde con las recomendaciones hechas por el Consejo de Europa. Westheimer y Kahne (2004) explican que la educación para la ciudadanía se aborda de un modo u otro en función del tipo de ciudadano que se pretenda promover. Por ejemplo, ellos identifican tres 'tipos ideales' de ciudadanos que se promueven en las escuelas: el ciudadano personalmente responsable (que cumple con los deberes estipulados en su comunidad), el ciudadano participativo (que se implica activamente en los asuntos cívicos y sociales, aunque sin análisis crítico) y el ciudadano orientado a la justicia social (que examina críticamente las realidades sociales insatisfactorias y lucha por su transformación).

Además, los procesos de toma de decisiones en las sociedades democráticas no suelen contribuir a la construcción de un ciudadano verdaderamente activo y universalista. Esto es debido a las deficiencias de las democracias representativas que provocan la desconfianza de la población en torno a los procesos políticos. Habría que recuperar, en este sentido, los componentes básicos de una auténtica ciudadanía democrática, los que tienen que ver con la participación activa y con el compromiso social (Torney-Purta y Barber, 2005). Claro que, trasladando esta idea al ámbito escolar, parece evidente que la educación para la ciudadanía no se consigue con una asignatura incluida en el plan de estudios de la educación obligatoria, sino que debe ser aprendida mediante el ejercicio de la participación y el pensamiento crítico en situaciones diversas (Cordero y Aguado, 2015), entre las que se deberían incluir acciones con repercusión real en la toma de decisiones en cuanto a la gestión de la escuela y de la comunidad.

Los resultados de las investigaciones de Santisteban y Pagès (2009) señalan que una alta proporción de estudiantes no conoce el sistema político ni sabe a qué administración pública debe dirigirse para solucionar problemas de la vida cotidiana, que consideran el voto como la forma más significativa de participación cuando sean mayores de edad y que su cultura cívica es, en general, pobre en cuanto a la resolución de conflictos, como, por ejemplo, conflictos de índole medioambiental.

Del mismo modo, García-Pérez y De Alba (2012) sostienen que los conocimientos políticos de la juventud son motivo de alarma y de preocupación, destacando que las carencias del alumnado en lo relativo a conceptos de política y democracia encuentran su causa en que la televisión es la principal fuente de suministro de las informaciones, promoviendo contenidos muy superficiales y aumentando el escepticismo por las formas de compromiso político.

Para superar, desde la educación, las carencias en cuanto a la democracia de la actualidad, se debe trabajar para la formación de una ciudadanía activa, crítica y participativa, lo cual significa asumir, según Cordero y Aguado (2015), unas premisas básicas que deberían caracterizar las propuestas formativas que pretendan facilitar el aprendizaje de la práctica ciudadana, más allá de unos contenidos conceptuales que provengan de un libro de texto. Los principios que deben ser requisito en las experiencias educativas de educación para la ciudadanía son, como exponen los autores mencionados, coherencia, praxis y participación, relación, justicia, cuidado, diversidad, diálogo, creatividad y crítica y transformación. Bajo estas premisas, una experiencia enriquecedora para formar a una ciudadanía crítica debe ser democrática, real y no excesivamente teórica (la ciudadanía se aprende ejerciéndola), que se desarrolle en relación con los iguales, valorando positivamente la diferencia y se base en principios de justicia y solidaridad, promoviendo la reflexión crítica de los hechos acaecidos.

Sin embargo, como afirman García-Pérez y De Alba (2012), la incorporación de la participación real a los procesos educativos no resulta fácil, pues el carácter de acción inherente a la actividad participativa entra en conflicto con la estructura escolar y, más concretamente, resulta poco compatible con el mundo de los contenidos académicos y de las estrategias metodológicas y recursos utilizados. En consecuencia, como se 
demuestra en los estudios de Tutiaux-Guillon (2009), los deseos del profesorado de formar alumnos en actitudes democráticas suelen ser divergentes con su propia práctica docente.

No obstante, de las experiencias reales que se basan en dinámicas de participación y de toma de decisiones por parte de niños y jóvenes, tanto a nivel escolar como comunitario, se pueden observar resultados positivos en diferentes áreas, desde la formación académica del alumnado hasta sus valores cívicos. A través de estas iniciativas, los alumnos aprenden a tomar decisiones y son conscientes de la importancia de participar en acciones sociales y comunitarias que lleven a cambios sistémicos para lograr una sociedad más equitativa y justa (Lucas y Delgado-Algarra, 2018).

Los procesos instructivos y experienciales llevados a cabo en la escuela, combinados con una serie de programas, estrategias y actos desarrollados en la propia ciudad, promueven que el alumnado vivencie las condiciones de construcción de una cultura urbana (Garcés, 2020) desde su entorno más cercano, fomentando actitudes críticas y participativas en el ámbito local sin perder el foco en la colaboración y participación global.

Los estudios que analizan algunas iniciativas democráticas y de participación, como es el caso de las Ecoescuelas o el Ilamado "Parlamento Joven", llevadas a cabo por investigadores de la Universidad de Sevilla, concluyen que la experiencia y el aprendizaje de quienes vivencian estas experiencias de forma directa se sienten muy comprometidos, tanto con el proyecto concreto como con las decisiones que afectan a la gestión de su entorno. Asimismo, resulta gratamente sorprendente la mejora de actitudes de muchos alumnos considerados académicamente como "malos alumnos", que, sin embargo, suelen responder a los requerimientos de estos programas (García-Pérez y De Alba, 2012). Además, en lo relativo a conocimientos políticos del alumnado, estos autores señalan que los estudiantes aumentan sus conocimientos cívicopolíticos y su interés en ellos en tanto que aumentan los procesos de participación en los que se implican y que las escuelas que practican un modelo democrático son más eficaces en la promoción de este tipo de conocimiento y compromiso.

Otro ejemplo de ello se puede encontrar en el análisis de dinámicas de presupuestos participativos y, particularmente, de presupuestos participativos escolares. Estos procesos democráticos de deliberación y toma de decisiones permiten el desarrollo de numerosas habilidades, como, por ejemplo, las de resolución de conflictos, capacitando a los participantes para transferir conocimientos, habilidades, disposiciones, valores y prácticas democráticas a otros espacios, haciendo conexiones entre el aprendizaje que ocurre en la escuela, en los procesos de democracia local, en el hogar y en otros espacios (Schugurensky, 2006; Cohen, Schugurensky y Wiek, 2015; Crum y Faydash 2018).

La educación democrática incluye no solo a la educación sobre democracia sino también a la educación para y en democracia. Siguiendo a Dewey (1916), una de las maneras más efectivas de aprender democracia es haciendo democracia. Solo cuando el alumnado experimenta procesos de participación real y con repercusión en las decisiones y en la gestión política de su entorno, comprende el efecto del activismo social y político, traducido en mejoras para la vida cotidiana y para el bien de la comunidad. Además, este tipo de tareas son idóneas para el fomento del pensamiento crítico, de nivel muy bajo en la juventud, según investigaciones de Santisteban y Pagès (2009), y para la formación de una ciudadanía que no asuma las decisiones políticas sin más, sino que reflexione sobre ellas y sea capaz de valorarlas en términos de justicia social y de preservación de los derechos humanos.

Estudios como el de Aguilar (2019), sobre prácticas ciberactivistas y sus distintas manifestaciones, como el videoactivismo, en contextos educativos formales propician en el alumnado un compromiso mayor frente a los problemas sociales y las posibles formas de intervenirlos, mediante acciones a medio y a largo plazo apoyadas en nuevos medios y tecnologías.

Es por esto que el presente artículo, que se enmarca en el Proyecto EPITEC "Educación Patrimonial para la inteligencia territorial y emocional de la ciudadanía. Análisis de buenas prácticas, diseño e intervención en la enseñanza obligatoria (código EDU2015-67953-P)", ha centrado nuestros intereses en el análisis de las propuestas y experiencias en torno a la educación para la ciudadanía, haciendo una revisión y caracterización de las mismas en cuatro revistas científicas y académicas españolas de los últimos diez años, con el fin tanto de conocer como de dar a conocer dichas prácticas y que puedan servir de modelo para centros escolares o para asociaciones juveniles de las diferentes comunidades.

\section{Marco metodológico}

Este artículo se llevó a cabo por medio de una investigación documental cualitativa que, siguiendo el método propuesto por Rodríguez-Medina, Gómez-Carrasco, López-Facal y Miralles-Martínez (2020), pretende 
describir los resultados obtenidos de una revisión bibliográfica y bibliométrica, teniendo como objeto conocer las investigaciones acerca de las experiencias desarrolladas en el aula que estén relacionadas con la educación ciudadana. Para ello, se eligieron cuatro revistas, debido a que son aquellas que mayor contenido abordan sobre didáctica de las Ciencias Sociales y porque, además, representan a varias universidades españolas comprometidas con esta disciplina (Extremadura, Valencia, Barcelona y Sevilla). Para dirigir la investigación se plantearon la pregunta de investigación y los objetivos explicitados en la tabla 1, que hacen referencia a conocer la prevalencia de proyectos o dinámicas educativas en torno a la educación ciudadana en España en base a lo expuesto en relevantes revistas académicas españolas en la temática y, por otro lado, a describir dichas experiencias didácticas, con el fin de caracterizar un modelo educativo que aporte beneficios al alumnado en lo relativo a la participación democrática dentro de la escuela y las distintas metodologías de enseñanza-aprendizaje que promuevan la formación de una ciudadanía reflexiva y crítica.

\section{Tabla 1}

Pregunta de investigación y objetivos del estudio.

\begin{tabular}{lll}
\hline \multicolumn{1}{c}{ Pregunta de investigación } & \multicolumn{1}{c}{ Objetivos } \\
\hline ¿Qué se ha publicado, en revistas académicas & $\bullet$ & Identificar lo que se ha escrito en los últimos \\
españolas, sobre innovación en las escuelas en & 10 años acerca de la educación ciudadana. \\
educación ciudadana? & $\bullet \quad \begin{array}{l}\text { Analizar las prácticas de educación } \\
\text { ciudadana descritas en esos trabajos }\end{array}$
\end{tabular}

Fuente: Elaboración propia.

\subsection{Proceso}

Para dar respuesta a la pregunta de investigación y a los objetivos planteados se desarrolló una revisión bibliográfica, con el fin de analizar los conocimientos y experiencias existentes acerca de la educación ciudadana en la última década en las cuatro revistas que se especifican a continuación. La elección de estas revistas no es arbitraria, sino que es resultante de una primera búsqueda de revistas académicas en acceso abierto y de relevancia en la temática en diferentes bases de datos internacionales y nacionales, entre las que destacamos Emerging Sources Citation Index (ESCI) y Dialnet. Se seleccionaron estas revistas por su dedicación casi en exclusiva a las investigaciones y experiencias en Didáctica de las Ciencias Sociales y por dedicar -en mayor o menor medida- atención al tema de la educación ciudadana en algunos de sus números monográficos. Estas son las siguientes:

\section{Investigación en la Escuela.}

Es una revista internacional de investigación e innovación escolar de la Universidad de Sevilla (US), que publica tres números al año. Se dirige prioritariamente al sector del profesorado y de los investigadores interesados en el estudio y mejora de los procesos de enseñanza y de formación del profesorado, desde una perspectiva abierta a la innovación, fundamentada y crítica. En su estructura actual, IE consta de dos grandes secciones: Investigaciones didácticas y Experiencias innovadoras. En cada una de ellas se incluyen artículos que pueden referirse a la enseñanza de materias escolares o a la formación inicial del profesorado.

\section{Revista de Investigación en Didáctica de las Ciencias Sociales.}

REIDICIS es una revista de investigación editada por la Asociación Universitaria de Profesorado de Didáctica de las Ciencias Sociales (AUPDCS) y patrocinada por la Universidad de Extremadura (UEX), con una periodicidad semestral. El principal objetivo de la publicación es la discusión abierta en Didáctica de las Ciencias Sociales, Geografía, Historia, Educación Patrimonial y Educación para la Ciudadanía. Con ello se persigue la difusión de trabajos de investigación reconocidos por su rigor científico y análisis crítico, en los siguientes ámbitos: formación del profesorado, currículo e innovación educativa a partir de los problemas de la enseñanza y el aprendizaje de las disciplinas sociales en contextos escolares.

\section{Didáctica de las Ciencias Experimentales y Sociales.}

Es una revista de la Universidad de Valencia (UV), cuya periodicidad es semestral, que se configura como una plataforma para difundir e intercambiar experiencias, investigaciones e innovaciones relacionadas con la didáctica de las Ciencias Experimentales y Sociales. Las temáticas principales de los artículos publicados 
giran en torno a la Física, la Química, la Biología y las Ciencias de la Tierra, la Educación Ambiental y Sostenibilidad, la Promoción y Educación para la Salud y la didáctica de la Historia y de la Geografía.

\section{Enseñanza de las Ciencias Sociales.}

Es una revista de la Universidad Autónoma de Barcelona (UAB) dedicada a la investigación en didáctica de la Geografía, la Historia y las Ciencias Sociales en general. Los objetivos que se propone son los dar a conocer los trabajos de investigación que se realizan en Didáctica de las Ciencias Sociales, profundizar en la base teórica y práctica de dichos trabajos y presentar nuevas líneas y nuevos temas de investigación en el ámbito de las Ciencias Sociales.

Para continuar con el proceso de selección de los artículos se hizo una clasificación con dos posibles vertientes: artículos que sí hablan sobre educación ciudadana y artículos de otras temáticas de la didáctica de las Ciencias Sociales. En la categoría de artículos que sí tratan sobre educación ciudadana se establece cada artículo que pertenece a cada una de las áreas: estudios conceptuales, teóricos y normativos; currículum y libro de textos; concepciones de alumnos y profesores; formación; propuestas; y experiencias concretas.

\subsection{Análisis de datos}

Con el fin de analizar los datos, se elaboró una ficha (tabla 2) para la sustracción de información, de manera que se pudiera realizar la clasificación de dicha información de todos los artículos de experiencias que fueron seleccionados, facilitando la recolección de los datos relacionados con la educación ciudadana.

\section{Tabla 2}

Ficha descriptiva de los artículos seleccionados.

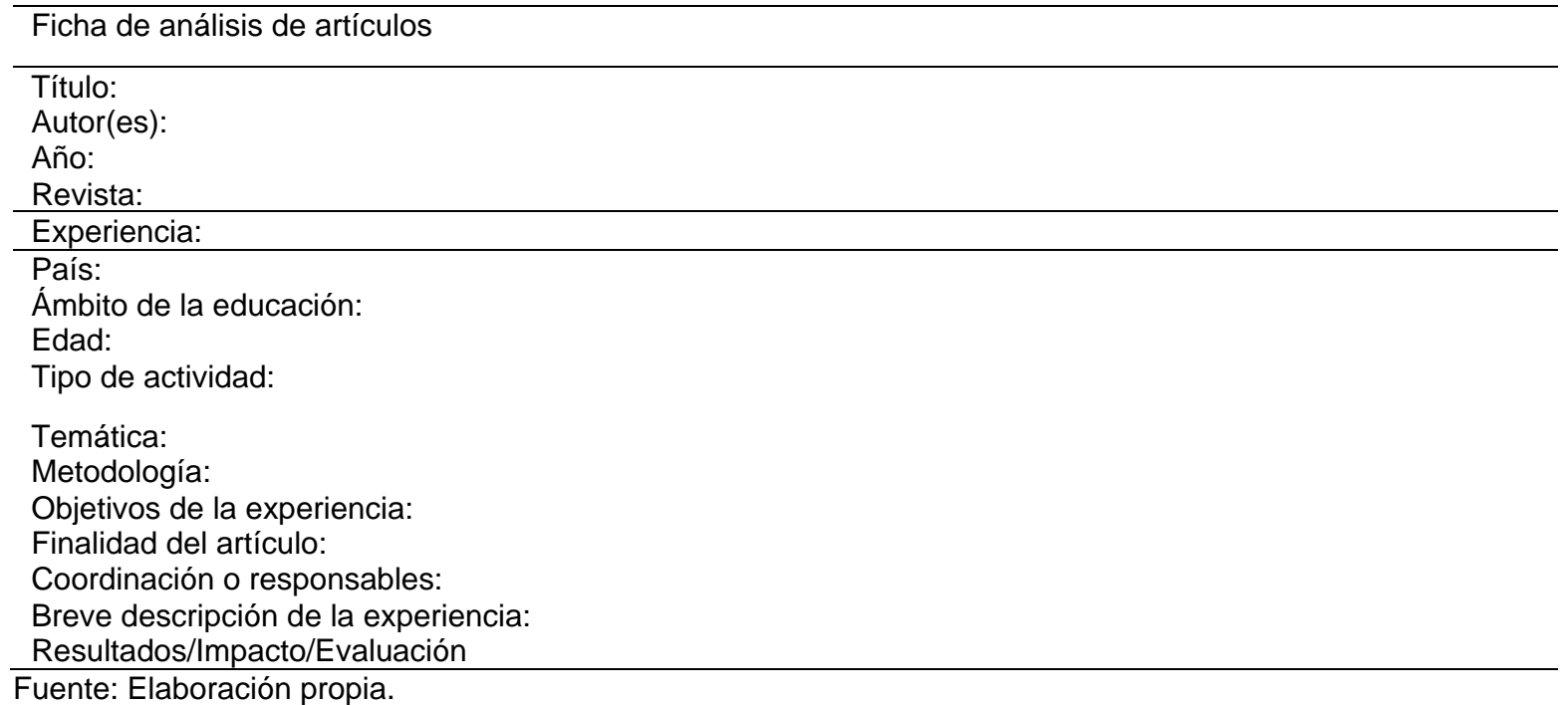

\section{Resultados}

Los resultados de esta búsqueda pormenorizada evidencian la escasez de artículos académicos que aborden en términos generales cuestiones acerca de la educación ciudadana:

1) En Investigación en la Escuela, de los 209 artículos publicados en los últimos 10 años, encontramos únicamente 25 artículos de EC.

2) En la Revista de Investigación en Didáctica de las Ciencias Sociales, de sus 24 artículos, 7 de los mismos tratan sobre EC.

3) En Didáctica de las Ciencias Experimentales y Sociales, de sus 139 artículos, solo 5 artículos giran en torno a la educación para la ciudadanía. 
4) En la última revista, Enseñanza de las Ciencias Sociales, encontramos 9 artículos de EC, de los 107 publicados en la última década.

En esta segunda fase de búsqueda, se analizaron un total de 479 artículos que abarcaban numerosas investigaciones y experiencias sobre la Didáctica de las Ciencias Sociales, en su mayoría. De estas publicaciones, encontramos 46 artículos que abordaban cuestiones en torno a la educación ciudadana y solamente 17 artículos describen experiencias o propuestas didácticas para trabajar sobre procesos democráticos y participación ciudadana en la escuela.

\subsection{Clasificación de las publicaciones}

Tras esta labor de búsqueda de artículos sobre EC en las cuatro revistas seleccionadas y teniendo en cuenta las palabras clave (educación ciudadana, participación, democracia, agencia, derechos/deberes, comunidad, aprendizaje-servicio, escuela, innovación, resolución de conflictos, convivencia, poder), se obtuvo la muestra de documentos presente en la tabla 3.

\section{Tabla 3}

Clasificación de artículos de educación ciudadana por temáticas.

\section{ARTÍCULOS DE EDUCACIÓN CIUDADANA POR TEMAS}

\begin{tabular}{lclcc}
\hline Temáticas & Revista Didáctica & Enseñanza de las & La investigación & Revista de \\
artículos EC & de las Ciencias & Ciencias Sociales & en la escuela & Investigación en \\
& $\begin{array}{c}\text { Experimentales y } \\
\text { Sociales }\end{array}$ & & & $\begin{array}{c}\text { Didáca de las } \\
\text { Ciencias Sociales }\end{array}$
\end{tabular}

\begin{tabular}{|c|c|c|c|c|}
\hline $\begin{array}{l}\text { Concepciones } \\
\text { Alumnos }\end{array}$ & 1 & 1 & 3 & 1 \\
\hline $\begin{array}{l}\text { Concepciones } \\
\text { Profesores }\end{array}$ & 0 & 0 & 1 & 1 \\
\hline Conceptual teórico & 0 & 4 & 3 & 0 \\
\hline $\begin{array}{l}\text { Currículum/Libro } \\
\text { de texto }\end{array}$ & 2 & 1 & 1 & 1 \\
\hline $\begin{array}{l}\text { Formación } \\
\text { docente }\end{array}$ & 1 & 1 & 2 & 3 \\
\hline Propuestas & 0 & 1 & 1 & 0 \\
\hline Experiencias & 1 & 1 & 14 & 1 \\
\hline TOTAL & 5 & 9 & 25 & 7 \\
\hline
\end{tabular}

Fuente: Elaboración propia.

Tal y como se expone en la tabla 3, existen pocos artículos académicos en España que sirvan de base teórica, conceptual y curricular para el tratamiento de diversos temas en torno a la educación ciudadana. No obstante, valoramos positivamente que la mayor parte de artículos encontrados se ocupan de propuestas didácticas y experiencias escolares y del análisis de las mismas, lo que demuestra que en las escuelas se están desarrollando ciertas prácticas educativas que fomentan la formación de una ciudadanía democrática, crítica y participativa en la comunidad a la que pertenece. 


\subsection{Propuestas y experiencias}

En la revisión de los 479 artículos, encontramos 17 que se relacionaban directamente con propuestas o experiencias en el área de la educación ciudadana, que son los siguientes:

1. Tonucci, D. (2009). ¿Se puede enseñar la participación? ¿Se puede enseñar la democracia? Investigación en la Escuela (68), 11-24. doi: 10.12795/IE.2009.i68.02

2. Haeberli, P. y Audifier, F. (2009). Participación y educación para la ciudadanía: el ejemplo de los consejos de clase. Investigación en la Escuela (68), 25-38. doi: 10.12795/IE.2009.668.03

3. Oraisón, M. (2009). Participación, escuela y ciudadanía: perspectiva crítica y praxis política. Investigación en la Escuela (68), 39-50. doi: 10.12795/IE.2009.i68.04

4. González, J.F. (2009). La formación para la participación ciudadana dentro y fuera de la escuela. Perspectivas desde Bogotá. Investigación en la Escuela (68), 63-72. doi: 10.12795/IE.2009.i68.06

5. De Alba, N. (2009). El parlamento joven: una experiencia de educación para la ciudadanía democrática. Investigación en la Escuela (68), 73-84. doi: 10.12795/IE.2009.i68.07

6. Ruiz, J. (2009). Espacios de participación ciudadana: Los Presupuestos Participativos de Sevilla y derivas educativas. Investigación en la Escuela (68), 85-100. doi: 10.12795/IE.2009.68. 08

7. Porlán, R. y De Alba, N. (2012). La escuela que queremos. Investigación en la Escuela (77), 5-12. doi: 10.12795/IE.2012.i77.01

8. Montalvo, S. (2012). La escuela que queremos, un lugar abierto al mundo. Investigación en la Escuela (77), 25-34. doi: 10.12795/IE.2012.i77.03

9. Martínez, M. (2012). Aprendiendo a decidir cuál es la escuela que queremos. Investigación en la Escuela (77), 35-42. doi: 10.12795/IE.2012.i77.04

10. Gordillo, I. (2012). La escuela que queremos: el germen de la transformación de nuestro patio. Investigación en la Escuela (77), 43-49. doi: 10.12795/IE.2012.i77.05

11. Salinas, M., López, F. y Masero, J. (2012). Aprender a ser felices. Investigación en la Escuela (77), 51-62. doi: 10.12795/IE.2012.i77.06

12. Pineda, J.A. (2012). Investigando mi escuela para mejorarla. Investigación en la Escuela (77), 63-74. doi: 10.12795/IE.2012.i77.07

13. Feito, R. (2014). Educar para la diversidad: el ejemplo de las "escuelas democráticas". Investigación en la Escuela (82), 7-17. doi: 10.12795/IE.2014.i82.01

14. Valderrama, R. y Solís, C. (2015). Investigación acción participativa como estrategia de transformación social y ambiental. Investigación en la Escuela (86), 49-59. doi: 10.12795/IE.2015.186.04

15. Parra, D. y Segarra, J.R. (2012). Celebraciones escolares, ¿fiestas cívicas? El tratamiento escolar del 9 d'Octubre y del Día de la Constitución en las aulas valencianas de Educación Primaria. Didáctica de las Ciencias Experimentales y Sociales (26), 19-34. doi: 10.7203/dces.26.1927

16. Galiana, V. (2018). La memoria democrática en las aulas de secundaria y bachillerato: balance de una experiencia didáctica. Didáctica de las Ciencias Experimentales y Sociales (34), 3-18. doi: $10.7203 /$ dces. 34.12048

17. Tosar, B. (2018). Literacidad crítica y enseñanza de las ciencias sociales en primaria: "Profe, las bolsas de plástico no son medusas". REIDICS. Revista de Investigación en Didáctica de las Ciencias Sociales (2), 4-19. doi: 10.17398/2531-0968.02.4

La gran mayoría de los 17 artículos (un 71\%) se enfoca en la descripción de experiencias orientadas a la participación democrática del alumnado y en el análisis del impacto de estas experiencias en los estudiantes, los docentes y el clima escolar. El resto de los artículos (29\%) se enfoca en iniciativas extraescolares que puedan influir en el espacio escolar y viceversa, procurando promover aprendizajes ciudadanos que trasciendan espacios específicos.

Desde el punto de vista de los objetivos de las iniciativas de educación ciudadana, 12 artículos mencionan el empoderamiento del alumnado para que sean portavoces de las necesidades que se generan en su entorno. Las otras experiencias se orientan a promover conciencia ciudadana ( 3 artículos) y a analizar iniciativas extraescolares que influyen en el espacio escolar e iniciativas escolares que trascienden al espacio no formal (2 artículos).

Desde el punto de vista de la metodología, 12 artículos usan metodologías cualitativas y acción participativa y los 5 artículos restantes incluyen metodologías mixtas. 
Por último, en cuanto a las conclusiones de los artículos, la mayoría de ellos enfatizan la necesidad de generar conciencia en el alumnado y la ciudadanía en general de cara a la escuela democrática y a la participación democrática.

\section{Discusión}

Los resultados de este estudio de revisión bibliométrica y bibliográfica sobre lo publicado acerca de experiencias innovadoras en educación ciudadana en los últimos diez años en las revistas académicas españolas seleccionadas han dejado una conclusión clara: la producción académica referida a este tipo de estudios sobre experiencias es realmente escasa. Aunque el total de estudios que tratan sobre la temática de "educación ciudadana" representa casi un $10 \%$ del total de investigaciones publicadas en dichas revistas, los artículos que analizan experiencias engloban un escaso $3 \%$.

Sin embargo, el porcentaje es aún más engañoso cuando vemos que de los 17 artículos sobre experiencias que hemos analizado, 10 corresponden a dos números monográficos (68 y 77) sobre experiencias en educación para la formación ciudadana que fueron publicados en la revista Investigación en la Escuela en los años 2009 y 2012. En el monográfico número 68 fueron publicados 6 artículos sobre experiencias, mientras que en el número 77 se publicaron 4 artículos. El total de experiencias publicadas en esta revista se eleva a 14, destacando sobremanera el interés en estos temas respecto a las demás y siendo la que más investigaciones sobre experiencias innovadoras ha publicado en temas de educación cívica. Las otras tres revistas apenas han publicado un artículo cada una sobre experiencias en la última década.

Esta situación tiene al menos dos posibles explicaciones, no necesariamente excluyentes. La primera es que se realizan pocas experiencias innovadoras en educación ciudadana en España, tanto en escuelas como en espacios extraescolares. Según esta tesis, no se puede estudiar lo que no existe y, en este sentido, si no se producen acciones no se pueden producir estudios sobre esas acciones. La segunda explicación es que los autores que publican en estas revistas académicas tienen poco interés en el estudio y análisis de la puesta en práctica de experiencias ejecutadas por los docentes en el área de educación ciudadana. Por diferentes razones, estos autores prefieren investigar aspectos teóricos, conceptuales y normativos relacionados con la Didáctica de las Ciencias Sociales, así como documentos curriculares, libros de texto, representaciones y concepciones de estudiantes y docentes, y formación docente inicial o en servicio.

De las 17 experiencias que hemos analizado, 13 han sido realizadas en España y las cuatro restantes en Italia, Suiza, Argentina y Colombia. La mayoría de estas experiencias (15) se han desarrollado en el ámbito de la educación formal, mientras que 2 de ellas se pusieron en marcha en ámbitos educativos informales. Los temas trabajados en esas experiencias han versado sobre aspectos diversos en relación a la educación cívica: fiestas e identidad, memoria histórica y democrática, construcción de una escuela democrática y participativa, acercamiento al juego político y democrático a través de los actores políticos, presupuestos participativos, acción participativa para la transformación socioambiental o literacidad crítica.

Destaca sobremanera el tema de la "construcción de una escuela democrática y participativa", con diversos estudios sobre experiencias de diversidad, inclusión del alumnado en la toma de decisiones, diseño y planificación de la escuela que se quiere y enfocada a ser felices. En este tipo de experiencias, cobraron una importancia crucial los referentes didácticos previos, focalizados en la figura del pedagogo Francesco Tonucci, siendo centro de atención de dos números monográficos de la revista Investigación en la Escuela.

Pero a pesar de lo que han reflejado estas 17 experiencias innovadoras, el escaso bagaje investigativo sobre la temática nos hace pensar que los temas de educación ciudadana se enfocan desde un modelo didáctico tradicional y, en menor medida, se participa de experiencias didácticas alternativas. Desde que en 2006 fuera establecida en España la asignatura de Educación para la Ciudadanía, con la Ley Orgánica de Educación del primer gobierno del socialista José Luis Rodríguez Zapatero, esta materia se ha enfocado más como una asignatura-batalla, como materia-piedra angular de los propósitos laicistas del PSOE y para confrontar a la asignatura de Religión. El déficit de la puesta en marcha de la asignatura, creemos, puede estar en que se presenta desde un punto de vista más teórico que práctico, dando más importancia a trabajarla desde el aula y en el aula con el libro de texto, de modo que los contenidos conceptuales jugaran un papel preponderante sobre los procedimentales y actitudinales; esto es, estudiarla sin ponerla en práctica, sin teatralizar el juego de la democracia y de la participación ciudadana, sin realizar un simulacro escolar, como han propuesto García-Pérez y De Alba (2012) y Muñoz (2012). Además, esta cuestión se habría acentuado tras la reforma educativa del Partido Popular con la LOMCE en 2013, pasando sus contenidos a ser transversales y a estudiarse como parte de diferentes asignaturas, muchas de ellas enfocadas mayoritariamente desde una metodología docente de carácter tradicional. 
En localidades y centros educativos de muchos países se han llevado a la práctica una gran variedad de experiencias de educación ciudadana que promueven no solo contenidos conceptuales sino también contenidos procedimentales (saber hacer) y actitudinales (valores) (Cohen, Schugurensky y Wiek, 2015; Pace, 2008; Zurita, 2010). Además de enseñar en qué consiste la participación democrática y transmitir conceptos básicos sobre civismo y democracia, muchos centros educativos y docentes individuales han practicado la materia, han jugado con la asignatura (Mancha Castro, 2019) y la han puesto en marcha tanto en centros escolares como en diversas comunidades locales y barrios o distritos. A través de estas experiencias, niños, jóvenes y adultos han vivido la participación ciudadana y el ejercicio de los principios de la democracia. Desde el punto de vista pedagógico, la idea que sustenta este abordaje es que una de las mejores maneras de aprender la democracia es haciendo democracia. Se trata de aprender haciendo, de rescatar el valor del aprendizaje a través de la experiencia. Esto no significa minimizar la importancia de los conceptos abstractos, sino simplemente de procurar un balance entre aprendizajes teóricos y prácticos.

Si algo podemos destacar de las investigaciones de experiencias que hemos analizado en las revistas que formaron la muestra es la transversalidad de los temas que se trataron. Esta cualidad da la posibilidad de integrarlos en trabajos por proyectos que trasciendan las materias tradicionales -incluida la propia educación para la ciudadanía- y hagan que el proceso de enseñanza-aprendizaje se genere en un clima de interés por parte del alumnado y partiendo de sus propias concepciones previas e intereses sobre temas relacionados con la democracia, la participación, la diversidad, la identidad, la política comunitaria y, en definitiva, todos los ámbitos que conforman el propio concepto de ciudadanía. Trabajar con competencias -y, en el caso de la educación ciudadana, hablamos especialmente de competencias sociales y cívicas (Santisteban, 2012)brinda la posibilidad de trabajar estas temáticas con otras asignaturas, particularmente en el área de las Ciencias Sociales.

\section{Conclusiones e implicaciones didácticas}

Teniendo en cuenta que, de los 479 artículos analizados, solamente 46 (menos del 10\%), se relacionan directamente con la educación para la ciudadanía y que tan solo un 3\% abordan propuestas pedagógicas innovadoras, podemos concluir que esta temática (sea tanto en el ámbito formal como fuera de la escuela) no tiene gran relevancia entre los académicos que han publicado en estas cuatro revistas. Esto es paradójico, pues la Ley Orgánica de Educación de España, impulsora de esta materia, señalaba que la educación debe promover el ejercicio de la ciudadanía democrática y la formación de ciudadanos autónomos, libres, críticos, responsables y comprometidos, recomendaba hacer el aprendizaje más atractivo y promocionar la ciudadanía activa, y planteaba que la práctica democrática debe inspirar el conjunto de la vida escolar y debe desarrollarse como parte de la educación en valores con carácter transversal a todas las actividades escolares. Por lo tanto, es relevante conocer más de lo que se está haciendo en el trabajo educativo cotidiano en torno a estos objetivos y contar con más análisis críticos sobre estas prácticas. Es también relevante saber más sobre las conexiones entre la materia "educación ciudadana democrática" con el resto de las intervenciones educativas.

En síntesis, en los casi 500 artículos que revisamos de las cuatro revistas, poco hemos encontrado sobre propuestas pedagógicas que formen ciudadanos reflexivos, críticos, comprometidos y competentes ya sea desde un ámbito formal o no formal. Aún más, en el contexto de los objetivos del desarrollo sostenible de las Naciones Unidas, todos los países signatarios se han comprometido a fortalecer la educación para la ciudadanía global, entendida esta como un nuevo modelo de ciudadanos comprometidos activamente en la consecución de un mundo más equitativo, sostenible, global, plural y solidario. Sin embargo, en los artículos que revisamos, prácticamente no aparece nada sobre proyectos educativos que promuevan la ciudadanía global.

Consideramos el sistema educativo como un elemento fundamental en la construcción de una ciudadanía responsable que desarrolle y ponga en práctica los valores democráticos de la modernidad (Latorre y Mérida, 2019). Así, siguiendo esta línea y teniendo en cuenta los aportes de las experiencias analizadas, surgen una serie de consideraciones para el diseño de prácticas educativas que promuevan una ciudadanía activa y democrática.

- Propuestas y/o proyectos significativos en los que se partan de los intereses, necesidades y experiencias previas del alumnado.

- Planteamiento de objetivos y contenidos en los que se desarrollen hábitos sociales y valores transversales. 
- Propuestas integrales que combinen reflexiones teóricas y actividades prácticas.

- Planteamientos participativos y atractivos que aseguren e inviten al compromiso de los diferentes destinatarios.

- Actividades sustentadas por el debate, el coloquio o diálogo, con el fin de promover la empatía y el pensamiento crítico.

Entre los posibles proyectos que se pueden implementar se incluyen los presupuestos participativos escolares, el parlamento joven, los consejos escolares, el decálogo de derechos y deberes del niño, la constitución escolar, role-playing sobre procesos democráticos adultos (como las elecciones), diseño de espacios escolares o actividades asamblearias. También se pueden considerar actividades encuadradas en el aprendizaje servicio como huertos escolares y comunitarios, reforestación del entorno próximo, campañas de sensibilización sobre el medio ambiente y su conservación, búsqueda de celebraciones internacionales del calendario de la ONU y reflexiones a partir de las mismas, lectura y análisis de la Declaración Universal de los Derechos Humanos, creación de cooperativas solidarias, organización de plataformas de crowdfunding, talleres de recogida de alimentos y juguetes para los más desfavorecidos, entre otras posibles. De este modo, la escuela, en su función primordial de educar a las generaciones futuras, ha de asumir con responsabilidad su capacidad de contribuir a la mejora de las comunidades a través del desarrollo de algunas de estas propuestas, con las que se pueden alcanzar de forma progresiva objetivos que, aunque ambiciosos, son necesarios en la sociedad actual.

Consideramos positivo que tanto en España como en otros países existen experiencias educativas que promueven la interiorización de aprendizajes ciudadanos funcionales y significativos a partir de la vivencia de experiencias y de ciclos continuos de reflexión y acción, que fomentan la participación en la sociedad, así como valores y actitudes de convivencia, cooperación, diálogo y justicia social. Estas propuestas tienen gran potencial para el desarrollo de valores y competencias democráticas y la investigación académica puede hacer más de lo que actualmente hace para contribuir al análisis y sistematización de dichas experiencias. Si de lo que se trata es de formar a ciudadanos y ciudadanas como personas con criterio propio, respetuosas, participativas y solidarias, que conozcan sus derechos y asuman sus deberes y sean capaces de ejercer la ciudadanía democrática de forma eficaz y responsable, la escuela debe transmitir conocimientos, actitudes y valores capaces de generar una cultura de solidaridad comprometida en la lucha contra la pobreza y la exclusión, así como con la promoción del desarrollo humano sostenible. Para ello, es preciso conocer más el estado de la cuestión y reflexionar teóricamente sobre los proyectos pedagógicos que se proponen estos objetivos, sean parte de la asignatura de educación ciudadana y democrática, de otras asignaturas, o de otros proyectos educativos. Aunque posiblemente la educación por sí misma no pueda democratizar sociedades, sí que puede formar personas más democráticas que construyan una sociedad más igualitaria, justa y plural.

\section{Referencias}

Aguilar Forero, N. (2019). Ciberactivismo y educación para la ciudadanía mundial: una investigación-acción participativa con dos experiencias educativas de Bogotá. Palabra Clave, 22(2), 1-31. doi: 10.5294/pacla.2019.22.2.10

Cohen, M., Schugurensky, D., y Wiek, A. (2015). Citizenship education through participatory budgeting: The case of Bioscience high school in Phoenix, Arizona. Curriculum and Teaching, 30 (2), 5-26. doi: 10.7459/ct/30.2.02

Cordero, C. y Aguado, T. (2015). Educación para la ciudadanía. Una asignatura a debate. Normativa, manuales y práctica escolar en España. Diálogo Andino - Revista de Historia, Geografía y Cultura Andina (47), 45-58. doi: 10.4067/S0719-26812015000200006

Cotton, K. (2001). Educating for Citizenship. Northwest Regional Educational Laboratory.

Crum, T. y Faydash, K. (2018). Youth Citizenship in Action: Evaluation of Participatory Budgeting in Schools Pilot. Chicago: University of Illinois.

Dewey, J. (1916). Democracy and education. An introduction to the philosophy. New York: Free Press.

Garcés, V.H. (2020). Alfabetizar en convivencia ciudadana. Una revisión documental de la educación ciudadana para la resolución de la violencia y el conflicto sociocultural. Sophia, 16(1), 4-18. doi: 0.18634/sophiaj.16v.1i.874

García-Pérez, F. F. (2009). Educar para la participación ciudadana. Un reto para la escuela del siglo XXI. Investigación en la Escuela 68, 5-10. doi: 10.12795/IE.2009.i68.01

García-Pérez, F. F. y De Alba, N. (2012). La educación para la participación ciudadana entre dos polos: el simulacro escolar y el compromiso social. En De Alba, N., García-Pérez, F. F. y Santisteban, A. (Eds.), Educar para la participación ciudadana en la enseñanza de las Ciencias Sociales, 1 (pp. 297-306). Sevilla: Díada.

Guanipa, L. F., Albites, J. L., Aldana, J. y Colina, F. (2019). Educación para la ciudadanía y la democracia: El equilibrio del poder. Iustitia Socialis. Revista Arbitrada de Ciencias Jurídicas, 4(6), 71-89. 
Latorre, G. y Mérida, R. (2019). La ciudad como contexto educativo: Desarrollo de la competencia cívica en las aulas infantiles y en el alumnado con necesidades de apoyo educativo. Foro de Educación, 17(27), 203-227. doi: http://dx.doi.org/10.14516/fde.649

Lerner, J. y Schugurensky, D. (2007). La dimensión educativa de la democracia local: el caso del presupuesto participativo. Temas y Debates, 13. doi: 10.35305/tyd.v0i13.140

Ley Orgánica 2/2006, de 3 de mayo, de Educación. Boletín Oficial del Estado, 4 de mayo de 2006, núm. 106. Ley Orgánica 8/2013, de 9 de diciembre, para la mejora de la calidad educativa. Boletín Oficial del Estado, 10 de diciembre de 2013, núm. 295.

Lucas, P. y Delgado-Algarra, E. (2018). Educación para una ciudadanía comprometida en la enseñanza de las Ciencias Sociales: ¿Qué piensa el alumnado de un profesor innovador sobre su aprendizaje? Didáctica de las Ciencias Experimentales y Sociales, 35, 3-16. doi: 10.7203/dces.35.12572

Mancha Castro, J. C. (2019). El franquismo en el aula. Una propuesta didáctica alternativa. Clío. History and History teaching (45), 268-300.

Morales Vargas, P. (2020). Educación ciudadana para la convivencia democrática y la interculturalidad en educación inicial. Revista Estudios En Educación, 3(4), 69-96.

Muñoz, C. (2012). El desarrollo de habilidades ciudadanas en la escuela. ¿Una educación para participar como gobernado o gobernante? En De Alba, N., García-Pérez, F. F. y Santisteban, A. (Eds.), Educar para la participación ciudadana en la enseñanza de las ciencias sociales, 1 (pp. 381-390). Sevilla: Díada.

Pace, J. (2008). Teaching for citizenship in 12th grade government classes. En Bixbi, J. y Pace, J. (Eds.), Educating democratic citizens in troubled times. Qualitative studies of current efforts (pp. 3-22). New York: SUNY.

Real Decreto 1105/2014, de 26 de diciembre, por el que se establece el currículo básico de la Educación Secundaria Obligatoria y del Bachillerato. Boletín Oficial del Estado, 3 de enero de 2015, núm. 3, Sección 1, pp. 169-546.

Real Decreto 126/2014, de 28 de febrero, por el que se establece el currículo básico de la Educación Primaria. Boletín Oficial del Estado, 1 de marzo de 2014, núm. 52.

Rodríguez-Medina, J., Gómez-Carrasco, C. J., López-Facal, R., Miralles-Martínez, P. (2020). Tendencias emergentes en la producción académica de educación histórica. Revista de Educación (389), 211-242.

Santisteban, A. (2012). La investigación sobre el desarrollo de la competencia social y ciudadana para una participación crítica. En De Alba, N., García-Pérez, F. F. y Santisteban, A. (Eds.), Educar para la participación ciudadana en la enseñanza de las ciencias sociales, 1 (pp. 277-286). Sevilla: Díada.

Santisteban, A. y Pagès, J. (2009). Una propuesta conceptual para la investigación en educación para la ciudadanía. Revista Educación y Pedagogía, 21 (53), 15-31.

Schugurensky, D. (2006). Strategies to elicit informal learning and tacit knowledge: Methodological notes from the field. Ontario: Institute for Studies in Education. University of Toronto.

Torney-Purta, J. y Barber, C. (2005). Democratic School Engagement and Civic Participation among European Adolescents: Analysis of Data from the IEA Civic Education Study. Journal of Social Science Education, Special Edition: European Year of Citizenship through Education.

Tutiaux-Guillon, N. (2009). Los IUFM y el nuevo máster. Íber: Didáctica de las Ciencias Sociales, Geografía e Historia, 61, 48-61.

Westheimer-Purta, J. y Kahne, J. (2004). What Kind of Citizen? The Politics of Educating for Democracy. American Educational Research Journal, 41 (2), 237-269.

Zurita, U. (2010). La educación para la vida democrática a través de la participación social: puntos de encuentro entre la escuela y la familia. Revista Interamericana de Educación para la Democracia, 3, (2), 172-194. 